\title{
Age as Indicator in the Selection of Surgery Modalities in Early Glottic Cancer
}

\author{
Jialing $\mathrm{Wu}$ (D)* \\ Kaiyun You* \\ Xingsheng Qiu* \\ Ting Shen \\ Juanjuan Song \\ Changlong Chen \\ Yanhui Jiang \\ Yimin Liu (D)
}

Department of Radiation Oncology, Sun Yat-sen Memorial Hospital, Sun Yat-sen University, Guangzhou, People's Republic of China

*These authors contributed equally to this work
Correspondence: Yimin Liu; Yanhui Jiang Department of Radiation Oncology, Sun Yat-sen Memorial Hospital, Sun Yat-sen University, 107 Yanjiang West Road, Yuexiu District, Guangzhou City,

Guangdong Province, People's Republic of China

Tel +86 I8922I825I3

Email liuyimin@mail.sysu.edu.cn;

jiangyh26@mail.sysu.edu.cn
Purpose: Local failure after endoscopic laryngeal surgery (ELS) for early glottic cancer mounts a challenge to researchers to investigate risk factors of recurrence. The present study was therefore designed to explore the prognostic factors in patients who underwent ELS for early glottic cancer.

Patients and Methods: We reviewed 328 patients with T1-2N0 glottic cancer who were treated with either ELS or open surgery between 2007 and 2018 at our institution. Survival, univariate and multivariate analyses were performed in different groups (ELS vs open surgery; $<65$ vs $\geq 65$ years).

Results: Age was discovered to be the independent prognostic factor of DFS for patients treated with ELS $(\mathrm{HR}=3.673, \mathrm{p}=0.003)$, but not for patients who underwent open surgery. Survival analysis performed on young patients $(<65$ years) showed that survival outcomes between different surgery modalities were significantly different (ELS vs open surgery: fiveyear DFS: 72.5 vs $84.7 \%, p=0.034)$. Univariate and multivariate analyses further confirmed the finding, whereas these results did not appear in old patients ( $\geq 65$ years).

Conclusion: Young patients $(<65$ years) treated with ELS had less favorable oncologic outcomes than those treated with open surgery. Young patients $(<65$ years) are advised to consider open surgery over ELS.

Keywords: early glottic cancer, age, recurrence, prognostic factor, surgery

\section{Introduction}

Laryngeal squamous cell carcinoma is one of the most common cancers in head and neck. ${ }^{1}$ Patients with laryngeal cancer are disproportionally males, and the most common subsite is glottis. ${ }^{2,3}$ Patients with glottic cancer often present with obvious hoarseness, which was conducive to early diagnosis and clinical intervention. Early glottic cancer, according to the extent of tumor and affected vocal cord, is subdivided into Tis, carcinoma in situ, T1a, confined to one cord, T1b, affects both cords, and T2, spreads beyond vocal cord or has cord mobility limited. For patients with early glottic cancer, organ-preserving treatment strategies such as radiotherapy and surgery are amenable. ${ }^{4}$ Five-year disease-free survival and overall survival of this population ranged between $74.9-92.8 \%$ and $80.0-96.0 \%$, respectively. ${ }^{4-8}$ The wide variety of survival outcomes is primarily attributed to the distinction of T stage, treatment modality, comorbidity, etc.

Surgical larynx-preserving modalities mainly included endoscopic laryngeal surgery (ELS), open cordectomy and partial laryngectomy, and surgery can be provided as a single treatment modality for patients with early glottic cancer. ${ }^{9}$ Although most patients with early glottic cancer can achieve a satisfctory local 
control rate and overall survival, recurrence is still a major issue and keeps deviating patients from normal life. There are abundant studies aiming to investigate the prognostic factors of patients treated with radiotherapy, ${ }^{5,10-12}$ while studies in regard to the relationship of factors and relapse of patients treated with surgery are comparatively rare.

In our institution, laryngeal cancer has been mainly and primarily managed by ELS (mainly transoral $\mathrm{CO}_{2}$ laser microsurgery) or partial laryngectomy. Since the superior vocal cord function can be achieved by ELS without the disruption of laryngeal surrounding anatomy, ELS has grown in popularity in recent years. We designed this study to find out the risk factors of recurrence for patients who underwent ELS for early glottic cancer, and we also investigated whether the risk factors were significant in patients treated with open laryngeal surgery.

\section{Patients and Methods}

We reviewed and collected the clinical information of patients who were diagnosed with early glottic cancer and treated with primary surgery between April 2007 and December 2018 in our institution. The study was conducted according to the guidelines of the Declaration of Helsinki, and approved by the ethics committee of our institution. The enrollment criteria of our study were: (1) biopsy-proven squamous cell carcinoma; (2) glottic cancer; (3) treated with ELS or open surgery; (4) stage I or II disease; (5) negative surgical margin; (6) previously untreated. In total, we extracted information of 915 patients who were diagnosed with laryngeal cancer. With regard to exclusion criteria, we first excluded patients diagnosed with supraglottic or subglottic cancer (174), then patients with stage III or IV disease (270), and then those histologically confirmed with sarcoma (25), as well as those with missing follow-up information (107) and those with positive surgical margin (11). Finally, we incorporated 328 patients in this study (312 males and 16 females; mean age, 64 years; range, 22-83 years). The staging of tumor-node-metastasis (TNM) of patients was on the grounds of preoperative and operative findings, as well as post-operative pathological results. TNM was determined according to the 7th edition of the American Joint Committee on Cancer (AJCC) for glottic cancer.

\section{Treatment of Patients}

Treatment of patients was based on the conditions and willingness of patients, and the recommendation of the multidisciplinary team. Patients treated with surgery were able to tolerate general anesthesia and laryngoscopy procedures. For tumor of $\mathrm{T} 1 \mathrm{a}, \mathrm{T} 1 \mathrm{~b}$ and selected $\mathrm{T} 2$ that could be exposed and visualized completely, ELS was preferred. For patients treated with ELS, a self-retaining laryngoscope was able to expose the whole lesion, and all of the tumor boundaries were included in the vision. Excision of the tumor was in accordance with the principles of surgical oncology. ELS was performed by a $\mathrm{CO}_{2}$ laser combined with the operating microscope. The quantity of $\mathrm{CO}_{2}$ laser power varied from 5 to $8 \mathrm{~W} .{ }^{13}$ Laryngeal endoscopic cordectomy was in line with the classification as well as the revision suggested by the European Laryngological Society. ${ }^{14}$ The modalities of open surgery mainly included cordectomy, vertical partial laryngectomy, and supracricoid partial laryngectomy. The greater part of open neck partial laryngectomies in our institution were vertical partial laryngectomy and supracricoid partial laryngectomy. Negative margins were achieved in all of the patients enrolled in this study.

\section{Follow-Up}

After the completion of treatment, patients were followed up closely until December 2020 or until death for those who died. Recurrence was determined as locoregional recurrence and distant metastasis. In our study, recurrence was defined as recurrence that happened at any time after primary surgery without a date limit, and usually required re-resection. Disease-free survival (DFS) was calculated as the time from the day of pathology-confirmed diagnosis to the day of recurrence or the date of last follow-up for those freed from recurrence; OS was the time from the day of pathology-confirmed diagnosis to the date of death of any causes or the date of last follow-up; Locoregional recurrence-free survival (LRFS) was the time from diagnosis to the day of locoregional recurrence or the date of last follow-up for those freed from locoregional recurrence.

\section{Statistical Analysis}

All statistical analyses were performed by using SPSS software (IBM, version 25.0). We first carried out univariate and multivariate analyses in patients treated with ELS, and then in patients treated with open laryngeal surgery. Afterward, we divided all the patients into two groups (age, $<65$ vs $\geq 65$ years), and performed survival, univariate and multivariate analyses in both groups. The relevance between classified variables and surgical modalities were analyzed by chi-square test. Factors including patient-related (age, gender, smoking history, drinking history), tumor-related ( $\mathrm{T}$ stage, pathological grade) and 
treatment-related (surgical modality) were analyzed. A two-sided P-value $\leq 0.05$ was viewed as statistical significance.

\section{Results}

\section{Univariate and Multivariate Analyses of DFS of Patients Treated with ELS}

Among 328 patients, 220 were treated with ELS, 206 were males $(93.6 \%)$ and 14 were females $(6.7 \%)$. The median age of this group was 61 years (range 22-83 years). T1 was in 176 patients and $\mathrm{T} 2$ was in 44 patients. One hundred and fifty-three patients had a history of smoking, 59 patients had a history of drinking, while 58 patients were both smokers and drinkers. Forty-five patients developed locoregional recurrence, among them, five developed cervical lymph nodes metastases. Three patients progressed with distant metastasis (lung). Seven patients were diagnosed with other cancers synchronously or asynchronously (including esophageal, breast, rectal, lung, liver cancer, and non-Hodgkin lymphoma).

Univariate and multivariate analyses of DFS were carried out in patients treated with ELS. The results showed that age $(<65$ vs $\geq 65$ years, hazard ratio (HR) of 3.673 , $95 \%$ confidence interval $(\mathrm{CI}): 1.557-8.664, \mathrm{p}=0.003)$ was an independent prognostic factor of DFS, while gender, $\mathrm{T}$ stage, pathological grade, smoking history and drinking history were not related with DFS (Table 1). We then proceeded to establish whether age played the same role in patients who were treated with open surgery.

\section{Univariate and Multivariate Analyses of DFS of Patients Treated with Open Laryngeal Surgery}

Among 328 patients, 108 underwent open laryngeal surgery; 106 were men (98.1\%) and two were women $(1.9 \%)$. The median age of this population was 62 years (range $31-$ 81 years). T1 was in 45 patients and T2 was in 63 patients. Eighty-two patients were heavy smokers, 40 patients were heavy drinkers, while 38 patients were both. Locoregional recurrence appeared in 15 patients, and three developed cervical lymph nodes metastases among them. One patient emerged with distant metastasis (bone). Seven patients were diagnosed with second cancer asynchronously (including esophageal cancer, lung cancer, pancreatic cancer, and nasopharyngeal cancer).

Univariate and multivariate analyses of DFS were conducted. The results showed that age, gender, $\mathrm{T}$ stage, pathological grade, smoking history and drinking history were not related with DFS of patients treated with open laryngeal surgery (Table 2).

Table I Univariate and Multivariate Analyses of DFS in Patients Treated with ELS

\begin{tabular}{|c|c|c|c|c|}
\hline \multirow[t]{2}{*}{ Variable } & \multicolumn{2}{|c|}{ Univariate } & \multicolumn{2}{|c|}{ Multivariate } \\
\hline & HR (95\% Cl) & $P$ value & HR (95\% Cl) & $P$ value \\
\hline \multicolumn{5}{|l|}{ Age, year } \\
\hline$<65$ vs $\geq 65$ & $3.673(1.557-8.664)$ & 0.003 & $3.67(1.557-8.664)$ & 0.003 \\
\hline \multicolumn{5}{|l|}{ Gender } \\
\hline Female vs male & $2.032(0.803-5.145)$ & 0.135 & \multicolumn{2}{|c|}{ NA } \\
\hline \multicolumn{5}{|l|}{$\mathbf{T}$ stage $^{\mathrm{a}}$} \\
\hline TI vs T2 & $0.733(0.372-1.445)$ & 0.370 & \multicolumn{2}{|c|}{ NA } \\
\hline \multicolumn{5}{|c|}{ Pathological grade } \\
\hline Good vs poor & $0.774(0.107-5.620)$ & 0.800 & \multicolumn{2}{|c|}{ NA } \\
\hline \multicolumn{5}{|l|}{ Smoking } \\
\hline Yes vs no & $1.338(0.679-2.636)$ & 0.400 & \multicolumn{2}{|c|}{ NA } \\
\hline \multicolumn{5}{|l|}{ Drinking } \\
\hline Yes vs no & $1.84 \mid(1.018-3.330)$ & 0.043 & \multicolumn{2}{|c|}{ NA } \\
\hline
\end{tabular}

Notes: ${ }^{a}$ Tumor-node-metastasis staging system proposed by the 7th edition American Joint Committee on Cancer (AJCC).

Abbreviations: ELS, endoscopic laryngeal surgery; DFS, disease-free survival; $\mathrm{Cl}$, confidence interval; $\mathrm{HR}$, hazard ratio. 
Table 2 Univariate and Multivariate Analyses of DFS in Patients Treated with Open Laryngeal Surgery

\begin{tabular}{|c|c|c|c|c|}
\hline \multirow[t]{2}{*}{ Variable } & \multicolumn{2}{|c|}{ Univariate } & \multicolumn{2}{|c|}{ Multivariate } \\
\hline & HR (95\% Cl) & $P$ value & HR (95\% Cl) & $P$ value \\
\hline \multicolumn{5}{|l|}{ Age, year } \\
\hline$<65$ vs $\geq 65$ & I.257(0.399-3.965) & 0.696 & \multicolumn{2}{|c|}{ NA } \\
\hline \multicolumn{5}{|l|}{ Gender } \\
\hline Female vs male & \multicolumn{2}{|c|}{ NA } & \multicolumn{2}{|c|}{ NA } \\
\hline \multicolumn{5}{|l|}{$\mathbf{T}$ stage $^{\mathbf{a}}$} \\
\hline TI vs T2 & I.654(0.562-4.87I) & 0.361 & \multicolumn{2}{|c|}{ NA } \\
\hline \multicolumn{5}{|c|}{ Pathological grade } \\
\hline Good vs poor & $0.392(0.087-1.759)$ & 0.222 & \multicolumn{2}{|c|}{ NA } \\
\hline \multicolumn{5}{|l|}{ Smoking } \\
\hline Yes vs no & $1.943(0.437-8.64 I)$ & 0.383 & \multicolumn{2}{|c|}{ NA } \\
\hline \multicolumn{5}{|l|}{ Drinking } \\
\hline Yes vs no & I.992(0.722-5.494) & 0.183 & \multicolumn{2}{|c|}{ NA } \\
\hline
\end{tabular}

Notes: ${ }^{\text {T}}$ Tumor-node-metastasis staging system proposed by the 7th edition American Joint Committee on Cancer (AJCC).

Abbreviations: DFS, disease-free survival; $\mathrm{Cl}$, confidence interval; $\mathrm{HR}$, hazard ratio.

Analyses above showed that age was an independent risk factor of DFS only for patients treated with ELS. The results illustrated that younger patients who underwent endoscopic procedures have an increased risk of recurrence. Furthermore, recurrence hazard of younger patients was the same as the older patients in open surgery group. Based on the results, we proposed a hypothesis that open laryngeal surgery can erase the effect of age. We then performed more analyses to confirm this hypothesis. The whole population was separated into two groups (age, $<65$ vs $\geq 65$ years), and survival, univariate and multivariate analyses were conducted.

\section{Survival, Univariate and Multivariate Analyses of Young Patients ( $<65$ Years)}

We first extracted young patients $(<65$ years $)$ from the whole study population, and 216 patients were eligible for analysis. The median follow-up time was 68 months (range $2-155$ months). The median age of this population was 57 years (range: 22-64 years). It was not a surprise to see that $\mathrm{T}$ stage was strongly correlated with surgery modality. Patients with $\mathrm{T} 2$ were predisposed to be treated with open laryngeal surgery, while patients with $\mathrm{T} 1$ were predisposed to be treated with ELS. However, the relationship between smoking history and surgery modality was unexpected. The baseline characteristics were listed in Table 3.

Survival analysis showed that survival outcomes (DFS and LRFS) between the two groups (ELS vs open surgery) were significantly different in young patients $(<65$ years). Five-year DFS was $72.5 \%$ in ELS group and $84.7 \%$ in open surgery group $(\mathrm{p}=0.034)$, and five-year LRFS was $73.1 \%$ in ELS group and $84.7 \%$ in open surgery group (p $=0.042$ ). However, the distribution of OS in two groups was similar (Table 4, Figure 1).

We further performed univariate and multivariate analyses in young patients, and the results demonstrated that surgery modality (ELS vs open surgery) was an independent prognostic factor of DFS, with hazard ratio of 2.027 (95\% CI: $1.040-3.953, p=0.038$ ). While gender, T stage, smoking and drinking history etc. were not related with recurrence (Table 5).

\section{Survival, Univariate and Multivariate Analyses of Old Patients ( $\geq 65$ Years)}

Afterward, we proceeded to investigate whether surgery modality exerts a prognostic influence on old patients. We then selected old patients ( $\geq 65$ years) from the whole study population, and 112 patients were selected and analyzed. The median follow-up time of this population was 
Table 3 Baseline Characteristics of Young Patients (< 65 Years)

\begin{tabular}{|l|c|c|c|}
\hline Variable & $\begin{array}{c}\text { ELS } \\
(\mathbf{N}=144)\end{array}$ & $\begin{array}{c}\text { Open Surgery } \\
(\mathbf{N}=\mathbf{7 2})\end{array}$ & P value \\
\hline $\begin{array}{l}\text { Gender } \\
\text { Male } \\
\text { Female }\end{array}$ & $\begin{array}{c}134 \\
10\end{array}$ & $\begin{array}{c}70 \\
2\end{array}$ & 0.345 \\
\hline $\begin{array}{c}\text { T stage }{ }^{\text {a }} \\
\text { T1 }\end{array}$ & 117 & 31 & 0.000 \\
T2 & 27 & 41 & \\
\hline $\begin{array}{l}\text { Pathological grade } \\
\text { Well differentiated }\end{array}$ & 141 & 68 & 0.225 \\
Poor differentiated & 3 & 4 & \\
\hline $\begin{array}{l}\text { Smoking history } \\
\text { Yes }\end{array}$ & 99 & 59 & 0.039 \\
No & 45 & 13 & \\
\hline $\begin{array}{l}\text { Drinking history } \\
\text { Yes }\end{array}$ & 42 & 27 & 0.216 \\
No & 102 & 45 & \\
\hline
\end{tabular}

Notes: ${ }^{a}$ Tumor-node-metastasis staging system proposed by the 7th edition American Joint Committee on Cancer (AJCC).

Abbreviation: ELS, endoscopic laryngeal surgery.

52.5 months (range 5-155 months). The median age was 70 years (range $65-83$ years). The results showed that the relationship between $\mathrm{T}$ stage and surgery modality was robust. The baseline characteristics of old patients $(\geq 65$ years) are listed in Table 6.

Kaplan-Meier analysis indicated that the survival distribution of the two groups (ELS vs open surgery) was not significantly different in old patients (Table 4). Further univariate and multivariate analyses conducted in old patients also put forward negative results (Table 7). These results validated our hypothesis that open surgery can erase the effect of age.

\section{Discussion}

Endoscopic laryngeal surgery (ELS, mainly transoral $\mathrm{CO}_{2}$ laser microsurgery), endowed with minimally invasive technology, is one of the valid surgical approaches for early glottic cancer. In contrast to conventional open laryngectomy and primary radiotherapy, the competitive edges of ELS include excellent local control, survival outcomes, and voice quality, as well as shorter hospital admission and lower mortality. ${ }^{15-18}$ Another benefit of ELS as reported by Moreau was that ELS facilitated the refinement of the TNM classification as certain carcinomas in situ were discovered to be invasive in
Table 4 Kaplan-Meier Analysis of Young and Old Patients

\begin{tabular}{|c|c|c|c|c|c|}
\hline \multicolumn{6}{|c|}{ Young Patients ( $<65$ Years) } \\
\hline \multirow[t]{2}{*}{ Group } & \multicolumn{2}{|c|}{ ELS $(N=144)$} & \multicolumn{2}{|c|}{ Open Surgery $(\mathrm{N}=72)$} & \multirow[t]{2}{*}{$P$ value } \\
\hline & 3-Year & 5-Year & 3-Year & 5-Year & \\
\hline DFS & $79.5 \%$ & $72.5 \%$ & $88.4 \%$ & $84.7 \%$ & 0.034 \\
\hline OS & $97.2 \%$ & $95.0 \%$ & $98.6 \%$ & $94.5 \%$ & 0.509 \\
\hline LRFS & $80.2 \%$ & $73.1 \%$ & $88.4 \%$ & $84.7 \%$ & 0.042 \\
\hline \multicolumn{6}{|c|}{ Old Patients ( $\geq 65$ Years) } \\
\hline \multirow[t]{2}{*}{ Group } & \multicolumn{2}{|c|}{ ELS $(\mathbf{N}=76)$} & \multicolumn{2}{|c|}{ Open Surgery $(\mathrm{N}=36)$} & $P$ value \\
\hline & 3-Year & 5-Year & 3-Year & 5-Year & \\
\hline DFS & $94.5 \%$ & $90.4 \%$ & $91.5 \%$ & $91.5 \%$ & 0.529 \\
\hline OS & $93.0 \%$ & $82.0 \%$ & $82.4 \%$ & $79.0 \%$ & 0.114 \\
\hline LRFS & $94.5 \%$ & $90.4 \%$ & $91.5 \%$ & $91.5 \%$ & 0.529 \\
\hline
\end{tabular}

Abbreviations: ELS, endoscopic laryngeal surgery; DFS, disease-free survival; OS, overall survival; LRFS, locoregional recurrence-free survival.

endoscopic excision. ${ }^{19}$ Moreover, studies showed that in respect of cost-utility, ELS prevailed over RT for T1 glottic cancer. ${ }^{20,21}$ However, the limitations of ELS lie in the additional perioperative risks of general anesthesia, as well as relatively poor voice outcomes compared with RT. $^{21,22}$ Based on the Surveillance, Epidemiology, and End Results (SEER) database, a study investigated the risks of second primary laryngeal cancer after initial surgery or RT, and showed a relative increased risk in the surgery group. However, the difference was not statistically significant. ${ }^{23}$ The theory of field cancerization illustrates that nearby organ tissue (e.g. aerodigestive tract mucosa) are normally exposed to the same carcinogens such as alcohol, tobacco and HPV infection, and thus possess similar precancerous lesions as the primary. The presence of field cancerization inevitably increases the event of locoregional recurrence and second primaries. ${ }^{24}$ Therefore, the single excision of the tumor performed by ELS may not be adequate to remove the lesion possessing similar genetic/epigenetic mutations.

Different from ELS, tissue resection in open surgery is complete and not confined by the extent of the tumor. ${ }^{9}$ Open partial laryngectomy is competent in obtaining excellent locoregional control without compromising the whole function of the larynx. A study showed that endoscopic excision succeeds in producing a decent survival outcome in most of cT2, but fails in tumor that upstaged as pT3 for involvement of the paraglottic space. ${ }^{25}$ The failure in exposing the lesion adequately with direct laryngoscopy 


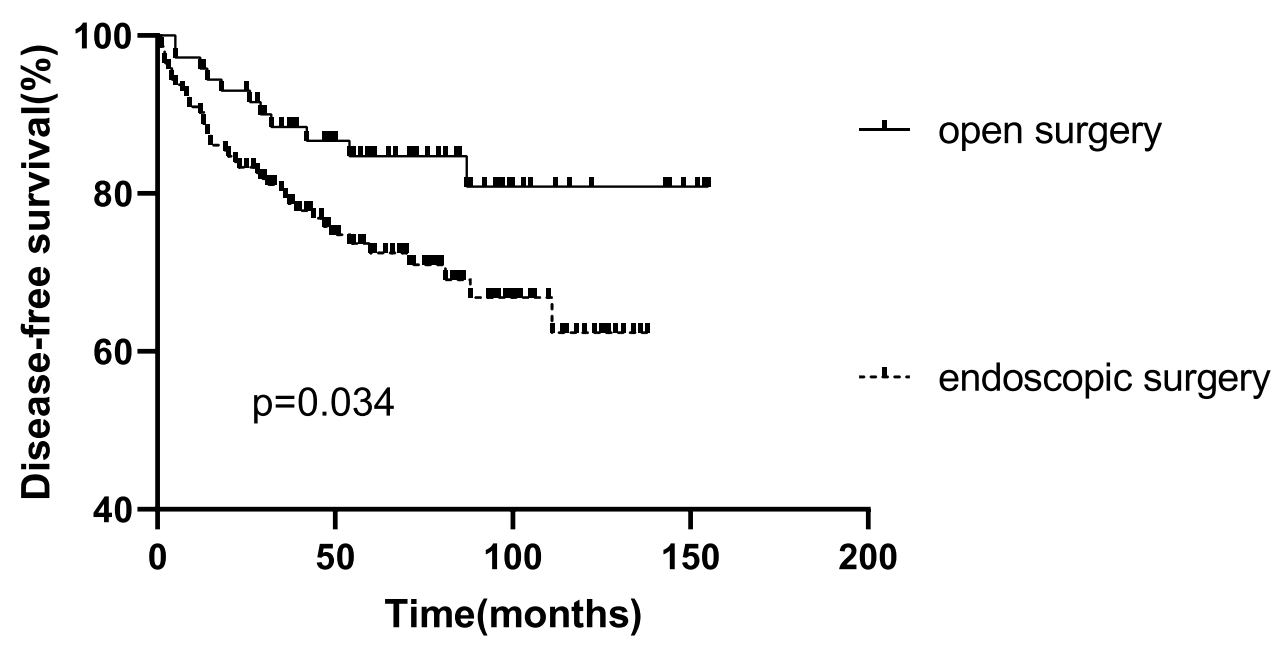

Figure I Kaplan-Meier curve of patients $<65$ years.

and the involvement of anterior commissure hinder the use of endoscopic surgery for T2 glottic cancer and support open partial laryngectomy. ${ }^{26,27}$ The outstanding oncological outcomes achieved by open partial surgery justify its role in selected cT2 patients. $^{28}$ However, the inherent qualities of open surgery such as prolonged recovery and hospitalization duration, higher mortality, requirement of tracheostomy, worse quality of life after operation and deteriorated voice mean that this treatment option is currently being supplanted. ${ }^{28}$ Open surgery is now reserved for treatment of advanced stage laryngeal carcinoma, locoregional failure of radiation or ELS for early stage cancer. Before being relegated by endoscopic procedures, open laryngectomy had been the standard treatment for early glottic cancer for almost half a century. ${ }^{29} \mathrm{We}$ should wonder, with the overwhelming preference toward ELS over open surgery, whether the pendulum may have swung too far in the direction of endoscopic approaches.

Table 5 Univariate and Multivariate Analyses of Young Patients (< 65 Years) of DFS

\begin{tabular}{|c|c|c|c|c|}
\hline \multirow[t]{2}{*}{ Variable } & \multicolumn{2}{|c|}{ Univariate } & \multicolumn{2}{|c|}{ Multivariate } \\
\hline & HR (95\% Cl) & $P$ value & HR (95\% Cl) & $P$ value \\
\hline \multicolumn{5}{|l|}{ Surgery modality } \\
\hline ELS vs open surgery & $2.027(1.040-3.953)$ & 0.038 & $2.027(1.040-3.953)$ & 0.038 \\
\hline \multicolumn{5}{|l|}{ Gender } \\
\hline Female vs male & $2.265(0.898-5.7 \mid 4)$ & 0.083 & \multicolumn{2}{|c|}{ NA } \\
\hline \multicolumn{5}{|l|}{$\mathbf{T}$ stage $^{\mathrm{a}}$} \\
\hline TI vs T2 & $0.962(0.532-1.739)$ & 0.898 & \multicolumn{2}{|c|}{ NA } \\
\hline \multicolumn{5}{|l|}{ Pathological grade } \\
\hline Good vs poor & $0.468(0.145-1.505)$ & 0.203 & \multicolumn{2}{|c|}{ NA } \\
\hline \multicolumn{5}{|l|}{ Smoking } \\
\hline Yes vs no & $1.227(0.629-2.394)$ & 0.548 & \multicolumn{2}{|c|}{ NA } \\
\hline \multicolumn{5}{|l|}{ Drinking } \\
\hline Yes vs no & $1.492(0.854-2.606)$ & 0.160 & \multicolumn{2}{|c|}{ NA } \\
\hline
\end{tabular}

Notes: ${ }^{a}$ Tumor-node-metastasis staging system proposed by the 7th edition American Joint Committee on Cancer (AJCC).

Abbreviations: DFS, disease-free survival; $\mathrm{Cl}$, confidence interval; $\mathrm{HR}$, hazard ratio. 
Table 6 Baseline Characteristics of Old Patients ( $\geq 65$ Years)

\begin{tabular}{|l|c|c|c|}
\hline Variable & $\begin{array}{c}\text { ELS } \\
(\mathbf{N}=\mathbf{7 6})\end{array}$ & $\begin{array}{c}\text { Open Surgery } \\
(\mathbf{N}=\mathbf{3 6})\end{array}$ & P value \\
\hline Gender & 72 & 36 & 0.303 \\
Male & 4 & 0 & \\
Female & 59 & 14 & 0.000 \\
\hline T stage & & \\
T1 & 17 & 22 & \\
T2 & 73 & 32 & 0.209 \\
\hline Pathological grade & 3 & 4 & \\
Well differentiated & & & 0.445 \\
Poor differentiated & 54 & 23 & \\
\hline Smoking history & 22 & 13 & 0.125 \\
Yes & 17 & 13 & \\
No & 59 & 23 & \\
\hline Drinking history & & & \\
Yes & & & \\
No & 549 & & \\
\hline
\end{tabular}

Notes: ${ }^{a}$ Tumor-node-metastasis staging system proposed by the 7th edition American Joint Committee on Cancer (AJCC).

Abbreviation: ELS, endoscopic laryngeal surgery.

Our study unveiled that age was an independent prognostic factor of DFS of patients treated with ELS, and not in patients treated with open laryngeal surgery.
Furthermore, survival analysis showed that recurrence tendencies of the two groups (ELS vs open surgery) significantly differed in young patients $(<65$ years), but did not differ in old patients ( $\geq 65$ years). Therefore, we drew an intriguing conclusion that patients with early glottic cancer who are under 65 years old may be justified in choosing open laryngeal surgery, for it had a more satisfactory oncology outcome in terms of DFS.

Incorporating 1616 patients with laryngeal squamous cell carcinoma, a cohort study indicated that old patients ( $>70$ years) had a significantly lower risk of recurrence in both glottic and supraglottic cancer, ${ }^{3}$ which was coincident with our study. Another study enrolled a total of 590 patients with early-intermediate (cTis-cT3) glottic cancer who received transoral laser microsurgery, and discovered that age $(<60 \mathrm{vs} \geq 60$ years) was significantly associated with recurrence-free survival, and the recurrence risk of young patients ( $<60$ years) was higher than old patients $(\geq$ 60 years) specifically. ${ }^{30}$ Analogously, Nomura et al. reported that relapse in the groups of T1b, T2, and T1-2 had a younger average age compared with the non-relapse groups, and age was independent of the other indicators and may be a useful prognostic factor. ${ }^{10}$ In contrast, a study of Rutkowski et al. suggested the opposite. ${ }^{31}$ The

Table 7 Univariate and Multivariate Analyses of Old Patients ( $\geq 65$ Years) of DFS

\begin{tabular}{|c|c|c|c|c|}
\hline \multirow[t]{2}{*}{ Variable } & \multicolumn{2}{|c|}{ Univariate } & \multicolumn{2}{|c|}{ Multivariate } \\
\hline & HR $(95 \% \mathrm{CI})$ & $P$ value & HR (95\% Cl) & $P$ value \\
\hline \multicolumn{5}{|l|}{ Surgery modality } \\
\hline ELS vs open surgery & $1.499(0.42 \mathrm{I}-5.338)$ & 0.532 & \multicolumn{2}{|c|}{ NA } \\
\hline \multicolumn{5}{|l|}{ Gender } \\
\hline Female vs male & \multicolumn{2}{|c|}{ NA } & \multicolumn{2}{|c|}{ NA } \\
\hline \multicolumn{5}{|l|}{ T stage ${ }^{a}$} \\
\hline TI vs T2 & $0.445(0.126-1.578)$ & 0.210 & \multicolumn{2}{|c|}{ NA } \\
\hline \multicolumn{5}{|l|}{ Pathological grade } \\
\hline Good vs poor & \multicolumn{2}{|c|}{ NA } & \multicolumn{2}{|c|}{ NA } \\
\hline \multicolumn{5}{|l|}{ Smoking } \\
\hline Yes vs no & $\mid .81 \mathrm{I}(0.383-8.558)$ & 0.454 & \multicolumn{2}{|c|}{ NA } \\
\hline \multicolumn{5}{|l|}{ Drinking } \\
\hline Yes vs no & $2.927(0.846-10.124)$ & 0.090 & \multicolumn{2}{|c|}{ NA } \\
\hline
\end{tabular}

Notes: ${ }^{\text {a }}$ Tumor-node-metastasis staging system proposed by the 7th edition American Joint Committee on Cancer (AJCC).

Abbreviations: DFS, disease-free survival; $\mathrm{Cl}$, confidence interval; $\mathrm{HR}$, hazard ratio. 
different result may be attributed to the fact that Rutkowski et al. enrolled only patients with $\mathrm{T} 2$ cancer.

It is important to mention that patients afflicted with early glottic cancer are not a single entity, but a quite heterogeneous group with great complexity and possibility, $^{32}$ and thus the treatment selection between open surgery and endoscopic approach of this population is intricate. Meticulous selection of the ideal patients who can benefit the most from ELS, and offering rigorous follow-up procedures to detect recurrence in time are recommended. More importantly, it is critical to cautiously evaluate the most suitable patients who can take the best advantage of open laryngeal surgery, and prevent the occurrence of a Pyrrhic victory. Although many recurrences at the glottis can be managed by endoscopic surgery repeatedly, ${ }^{33}$ and open laryngectomy for early glottic cancer is being gradually substituted by endoscopic procedures, primary open surgery may still be the better choice for particular patients. ${ }^{25}$ It is necessary to establish rigorous selection criteria on the grounds of tumor-related factors (size, extent, surgical margin status etc.), patientrelated factors (age, gender, expectation, general condition etc.) and treatment-related factors (cost, efficacy, relevant sequelae etc.), so as to deliver patients with early glottic cancer pleasant oncological and functional results.

Since surgery is the standard care for early glottic cancer in our institution, as well as the inclination of patients toward endoscopic procedures when provided both ELS and RT, we lacked patients who were treated with radiotherapy in particular. Our study therefore failed to evaluate the prognostic factors of patients treated with radiotherapy, as well as to investigate the comparison of radiotherapy and surgery in respect of oncologic and functional outcomes. Furthermore, due to the retrospective nature of our study and the lack of relevant medical records, other risk factors such as anterior commissure involvement, HPV status, tumor size and vocal cord immobility could not be evaluated.

\section{Conclusion}

The results of this study suggested that young patients with early glottic cancer should choose surgical alternatives (ELS vs open surgery) cautiously. Patients who are under 65 years are advised to choose open laryngectomy over endoscopic approaches, but more prospective studies are required to draw any concrete conclusions.

\section{Ethics Statement}

This study was conducted under the principle of the Institutional Review Board at Sun Yat-Sen Memorial Hospital, Sun Yat-Sen University (ethics approval number: SYSEC-KY-KS-2021-059). The present study was conducted retrospectively and did not interfere in the treatment of patients. The paper contains no patients' identifiable features, thus informed consents of the participants in the study were waived.

\section{Acknowledgments}

This study was funded by the Natural Science Foundation for Young Scholar of China (No. 82003071).

\section{Disclosure}

The authors report no conflicts of interest in this work.

\section{References}

1. Sung H, Ferlay J, Siegel RL, et al. Global cancer statistics 2020: GLOBOCAN estimates of incidence and mortality worldwide for 36 cancers in 185 countries. CA Cancer J Clin. 2021;71(3):209-249.

2. He Y, Liang D, Li D, et al. Incidence and mortality of laryngeal cancer in China, 2015. Chin $J$ Cancer Res. 2020;32(1):10-17. doi:10.21147/j.issn.1000-9604.2020.01.02

3. Brandstorp-Boesen J, Sorum Falk R, Folkvard Evensen J, et al. Risk of recurrence in laryngeal cancer. PLoS One. 2016;11(10):e0164068. doi:10.1371/journal.pone. 0164068

4. Shelan M, Anschuetz L, Schubert AD, et al. T1-2 glottic cancer treated with radiotherapy and/or surgery. Strahlenther Onkol. 2017;193(12):995-1004. doi:10.1007/s00066-017-1139-4

5. Elicin $\mathrm{O}$, Ermis $\mathrm{E}$, Oehler $\mathrm{C}$, et al. Influencing factors on radiotherapy outcome in stage I-II glottic larynx cancer-a Multicenter Study. Front Oncol. 2019;9:932. doi:10.3389/fonc.2019.00932

6. Chung SY, Kim KH, Keum KC, et al. Radiotherapy versus cordectomy in the management of early glottic cancer. Cancer Res Treat. 2018;50(1):156-163. doi:10.4143/crt.2016.503

7. Cetinayak O, Dogan E, Kuru A, et al. Outcome of early-stage glottic laryngeal carcinoma patients treated with radical radiotherapy using different techniques. J Oncol. 2019;2019:8640549. doi:10.1155/2019/ 8640549

8. Bledsoe TJ, Park HS, Stahl JM, et al. Hypofractionated radiotherapy for patients with early-stage glottic cancer: patterns of care and survival. J Natl Cancer Inst. 2017;109(10):10. doi:10.1093/jnci/ djx042

9. Smith RB. Surgery in the management of laryngeal and hypopharyngeal carcinoma. Int J Radiat Oncol Biol Phys. 2007;69(2):S28S30. doi:10.1016/j.ijrobp.2007.03.067

10. Nomura T, Ishikawa J, Ohki M, et al. Multifactorial analysis of local control and survival in patients with early glottic cancer. Laryngoscope. 2020;130(7):1701-1706. doi:10.1002/lary.28240

11. Sert F, Kaya I, Ozturk K, et al. Patterns of failure for early-stage glottic carcinoma: 10 years' experience in conformal radiotherapy era. $J$ Cancer Res Ther. 2019;15(3):576-581. doi:10.4103/jcrt.JCRT_692_17

12. Mucha-Malecka A, Chrostowska A, Urbanek K, et al. Prognostic factors in patients with $\mathrm{T} 1$ glottic cancer treated with radiotherapy. Strahlenther Onkol. 2019;195(9):792-804. doi:10.1007/s00066-01901481-2 
13. Liang F, Xiao Z, Chen $R$, et al. Transoral 980-nm/1470-nm dual-wavelength fiber laser microsurgery for early-stage glottic carcinoma. Oral Oncol. 2019;96:66-70. doi:10.1016/j. oraloncology.2019.07.007

14. Remacle M, Van Haverbeke C, Eckel H, et al. Proposal for revision of the European laryngological society classification of endoscopic cordectomies. Eur Arch Otorhinolaryngol. 2007;264(5):499-504. doi:10.1007/s00405-007-0279-z

15. Grant DG, Repanos C, Malpas G, et al. Transoral laser microsurgery for early laryngeal cancer. Expert Rev Anticancer Ther. 2010;10 (3):331-338. doi:10.1586/era.10.10

16. Valls-Mateus M, Ortega A, Blanch JL, et al. Long-term quality of life after transoral laser microsurgery for laryngeal carcinoma. J Surg Oncol. 2016;114(7):789-795. doi:10.1002/jso.24471

17. Canis M, Ihler F, Martin A, et al. Transoral laser microsurgery for T1a glottic cancer: review of 404 cases. Head Neck. 2015;37 (6):889-895. doi:10.1002/hed.23688

18. Mantsopoulos K, Psychogios G, Koch M, et al. Comparison of different surgical approaches in T2 glottic cancer. Head Neck. 2012;34(1):73-77. doi:10.1002/hed.21687

19. Moreau PR. Treatment of laryngeal carcinomas by laser endoscopic microsurgery. Laryngoscope. 2000;110(6):1000-1006. doi:10.1097/ 00005537-200006000-00022

20. Prettyjohns M, Winter S, Kerawala C, et al. Transoral laser microsurgery versus radiation therapy in the management of T1 and T2 laryngeal glottic carcinoma: which modality is cost-effective within the UK? Clin Otolaryngol. 2017;42(2):404-415. doi:10.1111/coa.12807

21. Higgins KM. What treatment for early-stage glottic carcinoma among adult patients: $\mathrm{CO} 2$ endolaryngeal laser excision versus standard fractionated external beam radiation is superior in terms of cost utility? Laryngoscope. 2011;121(1):116-134. doi:10.1002/lary.21226

22. Aaltonen L-M, Rautiainen N, Sellman J, et al. Voice quality after treatment of early vocal cord cancer: a randomized trial comparing laser surgery with radiation therapy. Int J Radiat Oncol Biol Phys. 2014;90(2):255-260. doi:10.1016/j.ijrobp.2014.06.032

23. Puram SV, Bhattacharyya N. Surgery versus radiation for T1 glottic carcinoma: second primary considerations. Laryngoscope. 2019;129 (12):2713-2715. doi:10.1002/lary.27854
24. Puram SV, Rocco JW. Molecular aspects of head and neck cancer therapy. Hematol Oncol Clin North Am. 2015;29(6):971-992. doi:10.1016/j.hoc.2015.07.003

25. Peretti G, Piazza C, Mensi MC, et al. Endoscopic treatment of cT2 glottic carcinoma: prognostic impact of different pT subcategories. Ann Otol Rhinol Laryngol. 2005;114(8):579-586. doi:10.1177/ 000348940511400801

26. DeSanto LW, Olsen KD. Early glottic cancer. Am J Otolaryngol. 1994;15(4):242-249. doi:10.1016/0196-0709(94)90090-6

27. Strong MS. Laser excision of carcinoma of the larynx. Laryngoscope. 1975;85(8):1286-1289. doi:10.1288/00005537-197508000-00003

28. Succo G, Crosetti E, Bertolin A, et al. Benefits and drawbacks of open partial horizontal laryngectomies, Part A: early- to intermediate-stage glottic carcinoma. Head Neck. 2016;38(Suppl S1):E333-E340. doi:10.1002/hed.23997

29. Silver CE, Beitler JJ, Shaha AR, et al. Current trends in initial management of laryngeal cancer: the declining use of open surgery. Eur Arch Otorhinolaryngol. 2009;266(9):1333-1352. doi:10.1007/ s00405-009-1028-2

30. Ansarin M, Cattaneo A, De Benedetto L, et al. Retrospective analysis of factors influencing oncologic outcome in 590 patients with early-intermediate glottic cancer treated by transoral laser microsurgery. Head Neck. 2017;39(1):71-81. doi:10.1002/hed.24534

31. Rutkowski T, Wygoda A, Skladowski K, et al. Prognostic role of tumor volume for radiotherapy outcome in patient with T2 laryngeal cancer. Strahlenther Onkol. 2013;189(10):861-866. doi:10.1007/ s00066-013-0411-5

32. Mannelli G, Comini LV, Santoro R, et al. T1 glottic cancer: does anterior commissure involvement worsen prognosis? Cancers 2020;12(6):6. doi:10.3390/cancers12061485

33. Mortuaire G, Francois J, Wiel E, et al. Local recurrence after CO2 laser cordectomy for early glottic carcinoma. Laryngoscope. 2006;116(1):101-105. doi:10.1097/01.mlg.0000184524.23282.74
Risk Management and Healthcare Policy

\section{Publish your work in this journal}

Risk Management and Healthcare Policy is an international, peerreviewed, open access journal focusing on all aspects of public health, policy, and preventative measures to promote good health and improve morbidity and mortality in the population. The journal welcomes submitted papers covering original research, basic science, clinical \& epidemiological studies, reviews and evaluations, guidelines, expert opinion and commentary, case reports and extended reports. The manuscript management system is completely online and includes a very quick and fair peer-review system, which is all easy to use. Visit http://www.dovepress.com/testimonials.php to read real quotes from published authors. 